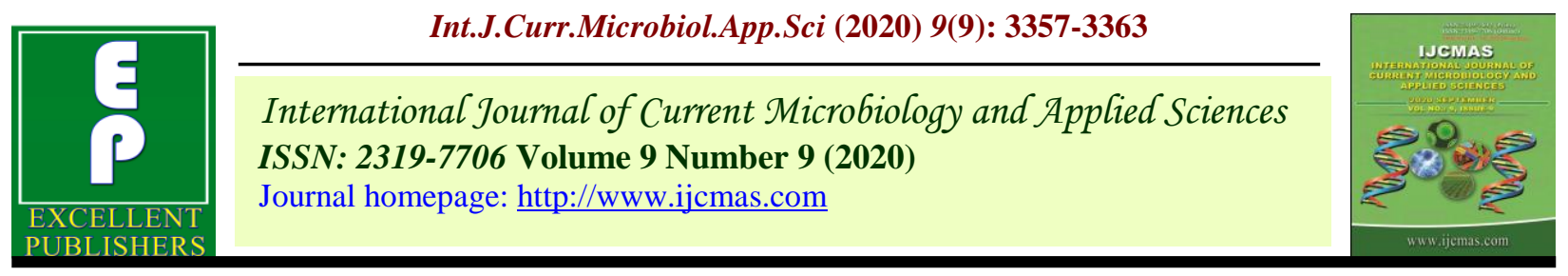

Original Research Article

https://doi.org/10.20546/ijcmas.2020.909.417

\title{
In vitro Antimicrobial Potential of Green Synthesised Silver Nano-Coated Cotton Gauzes
}

\author{
A. R. Gulnaz ${ }^{1 *}$, G. R. Chandrashekar ${ }^{2}$, R. M. Aravind ${ }^{3}$ and Shimya Vasu ${ }^{1}$ \\ ${ }^{1}$ Department of Biochemistry, ${ }^{2}$ Departmentof Operation Theatre Technology, ${ }^{3}$ Cauvery \\ Institute of Health Sciences, Cauvery Group of Educational Institutions Mysore, Karnataka \\ *Corresponding author
}

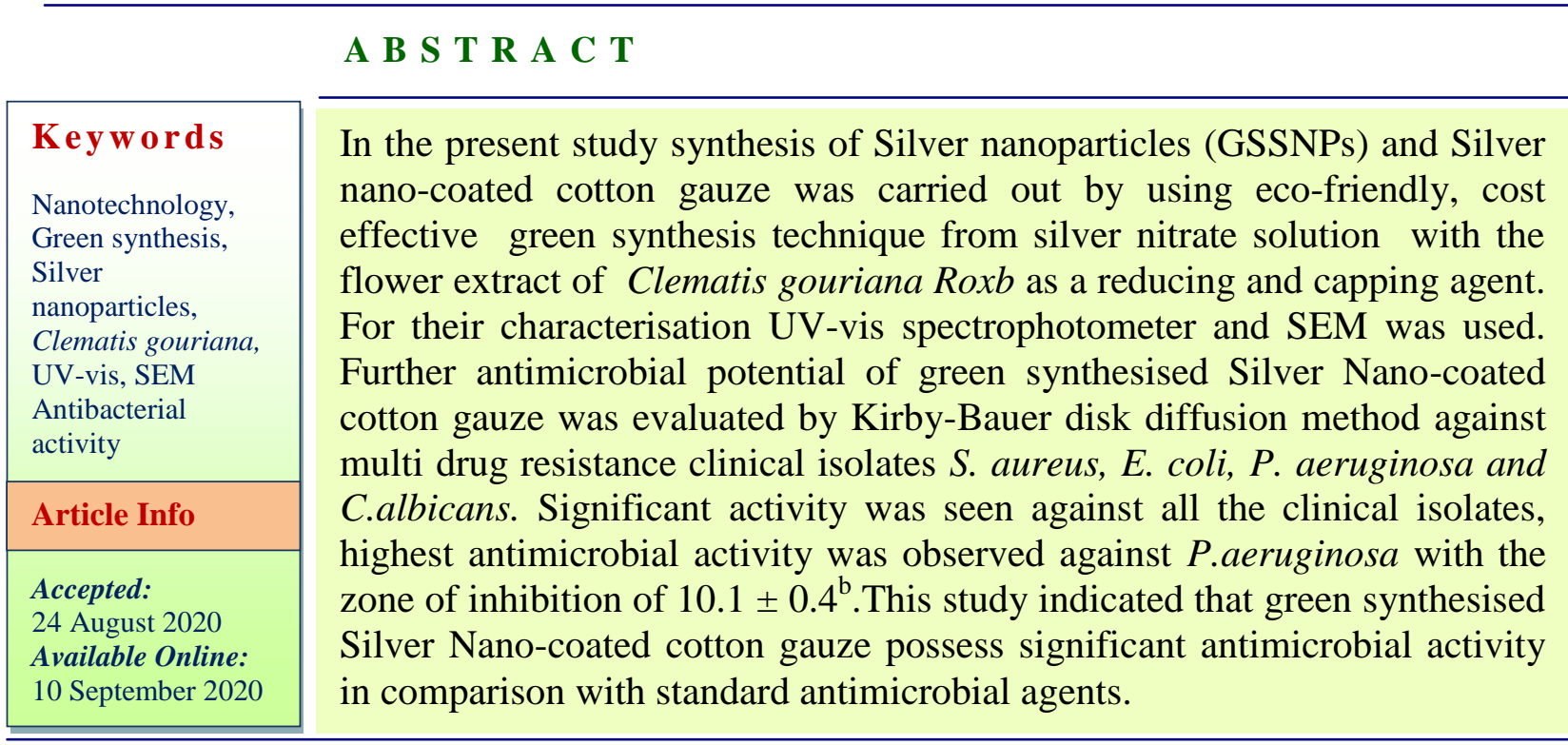

\section{Introduction}

Nanotechnology involves the study and application of extremely small particles which can be used across all the Science fields, such as chemistry, biology, physics, materials science, and engineering. Novel applications of nanoparticles and nanomaterials are growing rapidly on various fronts due to their completely new or advanced properties based on their size, distribution and morphology. It is rapidly leading to revolution in various fields such as health care, cosmetics, biomedical, food, feed, drug delivery, environment, health, mechanics, optics, chemical industries, electronics, space industries, energy science, catalysis, light emitters, single electron transistors, nonlinear optical devices, photo-electrochemical applications and many other ${ }^{1-3}$. It is expected that nanotechnology can change health care, and human life more profoundly compared to the past development. 
The metallic nanoparticles are considered to be most promising as they contain significant antimicrobial properties due to their large surface area to volume ratio, which is of much interest for researchers due to the growing microbial resistance against metal ions and antibiotics $^{2}$.Silver nanoparticles are of much importance in the field of nanotechnology which has gained interest because of their unique properties such as chemical stability, good conductivity, catalytic and its most important antibacterial, anti-viral, antifungal, anti-inflammatory activities. Silver functions as an antiseptic and displays a broad biocidal effect against microorganisms by disruption of their unicellular membrane thus disturbing their enzymatic activities. Indeed, unlike antibiotics, silver interferes with multiple components of bacterial cell structure and functions, making it less affected by specific micro-environmental variations ${ }^{4-6}$. In addition in the form of nanoparticles, silver plays a role in wound care and nanoparticles can also overcome existing drug resistance mechanisms ${ }^{7}$. As per the protocol silver nanoparticles are synthesized by chemical method using chemicals as reducing agents but this leads to various biological risks, to overcome this environment friendly biological green synthesis method has been found.

Development of green synthesis of nanoparticles is progressing as a key branch of nanotechnology where the use of bio components like microorganisms, phytocompounds of plant extracts such as proteins, polysaccharides, vitamins, glycosides, essential oils, phenolic-based chemicals etc are employed. Advantages of green synthesis over chemical and physical methods it is environment friendly, cost effective and easily scaled up for large scale syntheses of nanoparticles, furthermore there is no need to use high temperature, pressure, energy and toxic chemicals ${ }^{8}$. From the past few decades green synthesis of silver nanoparticles is emerging as a solution for the multi drug resistance microbes.

Clematis gouriana Roxb, is a woody climber belongs to the family Ranunculaceae. It is widely used in Ayurveda for the treatment of various ailments. Whole plant, stem and leaves are bitter, juice of the freshly crushed leaves and stems has a vesicant blistering action. Whole plant juice is used is to treat cold, headache and wound healing. Leaf extract is used to treat eczema, boils, itches, also applied to scabies, cuts and wounds, powdered leaves and flowers are used as insecticides. Roots decoction is given to treat stomach ache (CRC World Dictionary of Medicinal and Poisonous Plants). Aerial parts and roots contain a quaternary aporphine alkaloid, magno fluorine. The leaves contains a fungicide protoanemonin. Some researchers have proved its efficacy as antimicrobial agents $^{9}$, anti-ulcerative and hepato protective activity ${ }^{10}$. Our earlier studies on antiinflammatory activity $^{11}$ and antimicrobial activity against MDR oral pathogens ${ }^{12}$ has proved its candidature as an antiinflammatory and antimicrobial agent.

In the present study an attempt has been made to synthesize Silver nanoparticles and Silver nano-coated cotton gauze by using ecofriendly, cost effective green synthesis technique from silver nitrate solution with the flower extract of Clematis gouriana Roxbas a reducing and capping agent and further to evaluate the antibacterial effectiveness of Silver nano-coated cotton gauze against multidrug resistant clinical isolates.

\section{Materials and Methods}

\section{Preparation of plant extract}

Healthy disease free plant Clematis gouriana Roxb (CG) was collected, from Western 
Ghats. The plant was identified and authenticated at National Ayurveda Dietetics Research Institute Bangalore, (voucher no: RRCBI- 1194). Flowers are separated followed by repeated washing several times with tap water to remove the dust particles and then sun dried grinded to form powder, $1 \%$ of the CG flower extract was prepared by in deionized water in a conical flask and incubated for $30 \mathrm{~min}$ and Centrifuged for 30 minat $5000 \mathrm{rpm}$. The supernatant was used for the reduction of silver ions (Ag+) to silver nanoparticles (Ago).

\section{Green Synthesis of Silver Nanoparticles (GSSNPs)}

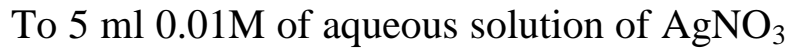
$5 \mathrm{ml} 1 \%$ of $\mathrm{CG}$ flower extract was added at ambient condition to react. After different time intervals, the color change of reaction mixture is observed from transparent yellow to dark brown which indicates the formation of GSSNPs. UV-vis spectral analysis was used to investigate the reduction mechanism of silver ion in to nanoparticles in solution. The GSSNPs solution was centrifuged and the excess liquid was removed by evaporation in a dryer to get black colored silver nanopowder.

UV-Vis analysis: was carried out for Characterization. The optical property of GSSNPs was determined by UV-Vis spectrophotometer. After adding the GC flower extract to the Silver nitrate solution, the spectra's were taken after $24 \mathrm{Hrs}$. between $300 \mathrm{~nm}$ to $700 \mathrm{~nm}$.

SEM Analysis: The morphological features of GSSNPs from CG flower extract were observed by Scanning Electron Microscope.

\section{Deposition of GSSNPs on cotton gauzes}

The cotton gauze purchased from local pharmacy was used. Deposition of Silver
Nanoparticles was done by micro encapsulation method. Sterile Gum acacia (natural polymer) was used as a wall material for coating. $10 \%$ gum acacia was prepared in hot water and allowed to stand on room temperature, to this $2.5 \mathrm{ml}$ GSSNPs were added with constant stirring. The mixture obtained was kept in freezing condition for 30 minutes to develop microcapsules. The cotton gauze was immersed in a microcapsule solution for 24 hours under dark condition. The immersed gauze was then padded and stored in sterile environment till further use.

\section{Physical characterization of GSSNPs- coated cotton gauzeby SEM}

SEM observation was done to study shape, size and surface area of the GSSNPscoated cotton gauze.

\begin{abstract}
Anti-Microbial Activity of GSSNPs-coated cotton gauzes: was carried out on four multi drug resistance microbes $S$. aureus, E. coli, $P$. aeruginosa and C.albicans. collected from clinical samples from Microbiology department, Cauvery Hospital Mysore, India, by Kirby-Bauer disk diffusion method. The nutrient agar plates for antibacterial activity and potato dextrose agar plate for antifungal activity were used. The test organisms were swabbed on to it, GSSNPs coated cotton gauze were cut into $1.5 \times 1.5 \mathrm{~cm}$ (length $\mathrm{x}$ breadth) and placed in the centre of a plate and incubated at $37^{\circ} \mathrm{C}$ for 24 hours. Plain gauzes as negative control and standard antibiotic disc were used as a positive controls. Zone of inhibition was recorded.
\end{abstract}

\section{Results and Discussion}

The preliminary phytochemical screening carried out had revealed the presence of many important compounds ${ }^{13}$. The presence of steroids, saponins, carbohydrates flavonoids etc acts as reducing agents capping agents 
which provide stability to silver nanoparticles. It was observed that solution of silver nitrate turned dark brown on addition of CG flower extract it indicated the formation of GSSNPs, while no color change was observed in the absence of CG flower extract (Fig. 1). In the UV-Vis spectrum a single, strong and broad Surface plasm on resonance (SPR) peak was observed at $414 \mathrm{~nm}$ that confirmed GSSNPs (Fig. 2).

Table.1 Antimicrobial activity of GSSNPs -coated cotton gauzes

\begin{tabular}{|l|c|c|c|c|}
\hline Antibiotic disc & S. aureus & E. coli & P. aeruginosa & C.albicans \\
\hline Clavulanic acid 30 & R & R & R & R \\
\hline Cefixime & $07 \pm 7.10$ & R & R & R \\
\hline Amikacin & $08 \pm 0.46^{c}$ & $\mathbf{R}$ & $\mathbf{R}$ & $\mathbf{R}$ \\
\hline Tetracyclin & $\mathbf{R}$ & $07 \pm 7.10$ & $\mathbf{R}$ & $\mathbf{R}$ \\
\hline $\begin{array}{l}\text { GSSNP-coated } \\
\text { cotton gauzes }\end{array}$ & $07 \pm 0.46^{\mathrm{c}}$ & $7 \pm 0.1^{\mathrm{a}}$ & $10.1 \pm 0.4^{\mathrm{b}}$ & $08 \pm 0.2^{\mathrm{a}}$ \\
\hline
\end{tabular}

Fig.1 Green Synthesis of Silver nanoparticles

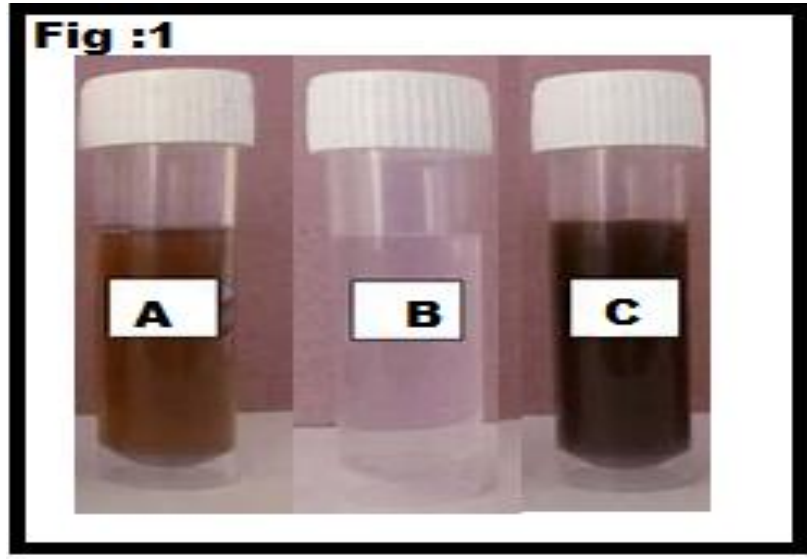

(A) CG flower extract. (B) Silver Nitrate Solution.

(C) Silver Nanoparticle

Fig.2 UV- VIS spectroscopy for GSSNPs

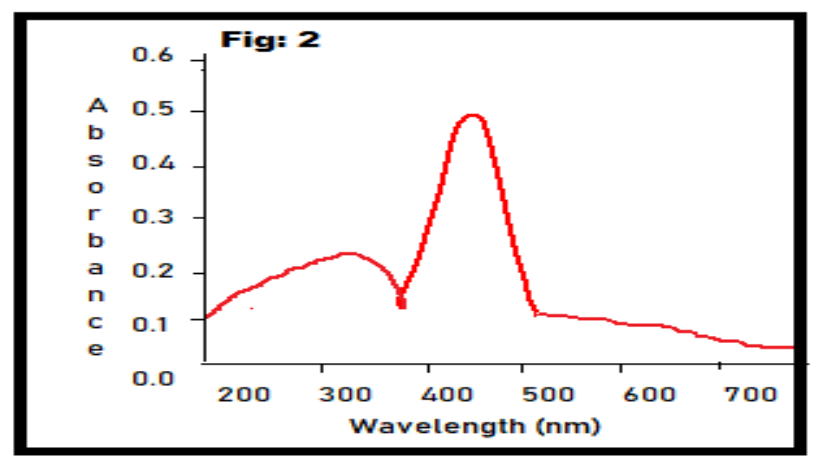


Fig.3 GSSNPs SEM-analysis

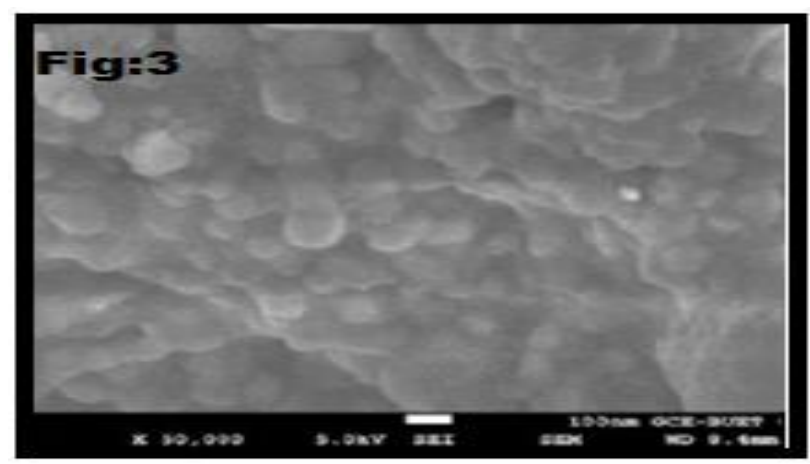

Fig.4 GSSNPs-coated cotton gauze

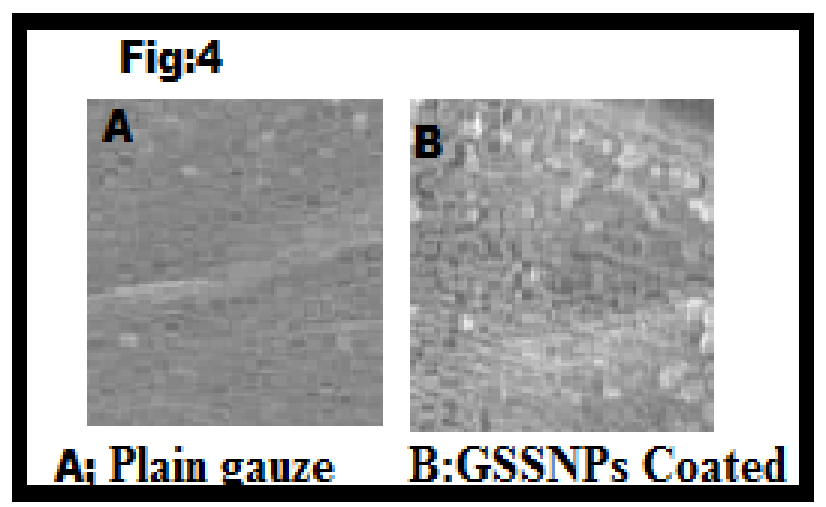

Figure 3 shows the SEM images of the GSSNPs. SEM images showed that majority of the GSSNPs are spherical in shape with smooth surface compactly arranged and well dispersed. The average particle size was found around $6.45 \mathrm{~nm}$.

\section{Physical characterization of GSSNPs- coated cotton gauze by SEM}

Figure 4 shows the SEM images of the GSSNPs coated cotton gauze

\section{Antimicrobial Activity of GSSNPs -coated cotton gauzes}

Significant activity was seen against all the clinical isolates, highest antimicrobial activity was observed against P.aeruginosa with the zone of inhibition of $10.1 \pm 0.4^{\mathrm{b}}$ (Table 1 ).
It is difficult to combat with multi-resistant pathogens due to antigenic shifts and/or drifts. This resistance to medication by pathogens has become a stern problem in public health hence there is a strong requirement to develop new bactericides and virucides. Silver is having a long history of use as an antiseptic and disinfectant and is able to interact with disulphide bonds of the glycoprotein/ protein contents of microorganisms such as viruses, bacteria and fungi. Both silver nanoparticles and silver ions can change the three dimensional structure of proteins by interfering with disulphide bonds and block the functional operations of the microorganism. Green synthesized silver nanoparticles offers numerous benefits of eco-friendly and compatibility for pharmaceutical and other biomedical applications as they do not use toxic chemicals for the synthesis protocol. 
It could be concluded that cotton gauze coated with green synthesized silver nanoparticles with the flower of extract of Clematis gouriana has significant in vitro antimicrobial activity against multidrug resistance clinical isolates, hence the potential therapeutic use of green synthesized silver nanoparticles opens a door for a new range of antibacterial agents.

\section{References}

1. Korbekandi H, Iravani S. Silver nanoparticles, the delivery of nanoparticles. In: Hashim Abbass A., editor, ISBN: 978-95351-0615-9, InTech; 2012.

2. Khalil KA, Fouad H, Elsarnagawy T, Almajhdi FN. Preparation and characterization of electrospun PLGA silvercomposite nanofibers for biomedical applications. Int $\mathrm{J}$ ElectrochemSci 2013; 8:3483-93.

3. Kaviya SSJ, Viswanathan B. Green synthesis of silver nanoparticles using Polyalthia longifolia leaf extract alongwith D-sorbitol. J Nanotech 2011:1-5.

4. Bjarnsholt, T., Kirketerp-Moller, K., Kristiansen, S., Phipps, R., Nielsen, A.K., Jensen, P.O., Hoiby, N., Givskov, M. Silver against Pseudomonas aeruginosa biofilms. APMIS 2007, 115, 921-928.

5. Lansdown, A.B. Silver I: Its antibacterial properties and mechanism of action. J.Wound Care 2002, 11, 125-130.

6. Kostenko, V., Lyczak, J., Turner, K., Martinuzzi, R.J. Impact of SilverContaining Wound Dressings on Bacterial Biofilm Viability and Susceptibility to Antibiotics during Prolonged Treatment. Antimicrob. Agents Chemother. 2010, 54, 51205131.

7. Pelgrift, R.Y., Friedman, A.J. Nanotechnology as a therapeutic tool to combat microbial resistance. Adv. DrugDeliv. Rev. 2013, 65, 1803-1815.

8. Shamim N and Sharma V.K Green Nanotechnology: Development of Nanomaterial for Environmental and Energy Applications. Chap 12 in Sustainable Nanotechnology and the Environment: Advances and Achievements, 2013.Vol. 1124. pp 201229. American Chemical Society. ISBN13: 9780841227842.

9. Sankar Narayan Sinha., Phytochemical analysis and antibacterial activity of Clematis gouriana Roxb. Int.J.Pharm.Anal. Vol: 2 Issue:5 Page: 399-401.

10. Gulnaz, A.R., Anusuya, M.R., Husna Almas, Gururaj, R., Savitha, G., In vitro Anti-Inflammatory activity and Phytochemical analysis of ethanolic extract of Clematis Gouriana Roxb leaf (UGC sponsored national level conference at Sarada Vilas College Mysore Recent Trends in Bioorganic chemistry and its applications to societyRTBAS 2014 ISBN :978-81-930115-0-8. Page: 46-50.

11. Kulkarni A. S., VijayaRaghavan C., Patil D.M., Polyherbal mixture Protects against Ethanol- Induced Gastric Ulcers and liver necrosis in Rats. Journal of Pharmacy Research 2012, 5(8), 41694171.

12. Yogesh, B.G., P.Sheshadri, N. Anees Ahmed, A.R. Gulnaz and Vatsala, R. 2016. Phytochemical Analysis and Antimicrobial Activity of Different Extracts of Clematis gouriana. Roxb Flower on Multi Drug Resistance oral Pathogens. Int.J.Curr.Microbiol.App.Sci. 5(3: 686-691.

13. Cavanagh, M.H., Burrell, R.E., Nadworny, P.L. Evaluating antimicrobial efficacy of new commercially available silver dressings. Int. Wound J. 2010, 7, 394-405. 
14. Knetsch, M.L.W., Koole, L.H. New Strategies in the Development of Antimicrobial Coatings: The Example of
Increasing Usage of Silver and Silver Nanoparticles. Polymers 2011, 3, 340366.

\section{How to cite this article:}

Gulnaz, A. R., G. R. Chandrashekar, R. M. Aravind and Shimya Vasu. 2020. In vitro Antimicrobial Potential of Green Synthesised Silver Nano-Coated Cotton Gauzes. Int.J.Curr.Microbiol.App.Sci. 9(09): 3357-3363. doi: https://doi.org/10.20546/ijcmas.2020.909.417 